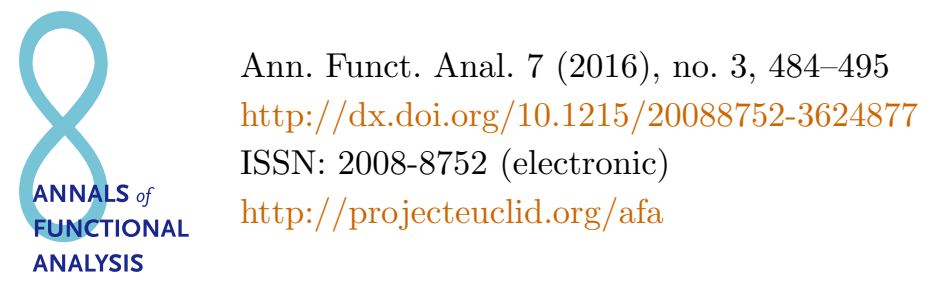

\title{
INTERPOLATION OF NONCOMMUTATIVE QUASIMARTINGALE SPACES
}

\author{
CONGBIAN MA* and YOULIANG HOU
}

Communicated by K. Zhu

\begin{abstract}
Let $\widehat{L}_{p}(\mathcal{M})$ be the space of bounded $L_{p}(\mathcal{M})$-quasimartingales. We prove that, with equivalent norms, $\left(\widehat{L}_{p_{0}}(\mathcal{M}), \widehat{L}_{p_{1}}(\mathcal{M})\right)_{\theta, p}=\widehat{L}_{p}(\mathcal{M})$, where $1<p_{0}, p_{1} \leq \infty, 1<\theta<1$, and $\frac{1}{p}=\frac{1-\theta}{p_{0}}+\frac{\theta}{p_{1}}$. We also prove that, for $1<p<$ $q<\infty,\left(\widehat{\mathrm{BMO}}^{c}(\mathcal{M}), \widehat{\mathcal{H}}_{p}^{c}(\mathcal{M})\right)_{\frac{p}{q}, q}=\widehat{\mathcal{H}}_{q}^{c}(\mathcal{M})$ and $\left(\widehat{\mathrm{BMO}}^{r}(\mathcal{M}), \widehat{\mathcal{H}}_{p}^{r}(\mathcal{M})\right)_{\frac{p}{q}, q}=$ $\widehat{\mathcal{H}}_{q}^{r}(\mathcal{M})$, where $\widehat{\mathcal{H}}_{p}(\mathcal{M})$ and $\widehat{\mathrm{BMO}}(\mathcal{M})$ are, respectively, the Hardy space and the bounded mean oscillation space of noncommutative quasimartingales.
\end{abstract}

\section{IntRoduction}

Inspired by quantum mechanics and probability, noncommutative probability has become an independent field of mathematical research. Today, many of the classical martingale inequalities have been transferred to the noncommutative setting. These include, in particular, the Doob maximal inequality, the BurkholderGundy inequality, several weak-type $(1,1)$ inequalities, and the Gundy decomposition.

As for the interpolation between the spaces of noncommutative martingales, we recall the formula on real and complex interpolation of noncommutative

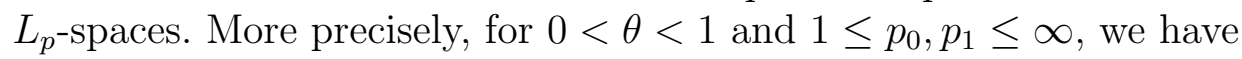

$$
\left(L_{p_{0}}(\mathcal{M}), L_{p_{1}}(\mathcal{M})\right)_{\theta, p}=L_{p}(\mathcal{M}) \quad \text { and } \quad\left(L_{p_{0}}(\mathcal{M}), L_{p_{1}}(\mathcal{M})\right)_{\theta}=L_{p}(\mathcal{M})
$$

Copyright 2016 by the Tusi Mathematical Research Group.

Received Dec. 2, 2015; Accepted Feb. 3, 2016.

${ }^{*}$ Corresponding author.

2010 Mathematics Subject Classification. Primary 46L53; Secondary 46L52, 60 G42.

Keywords. noncommutative quasimartingale, interpolation space, Hardy space. 


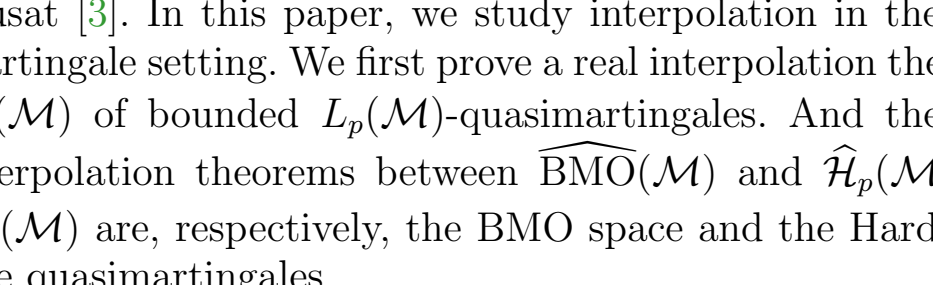

\title{
INTERPOLATION OF NONCOMMUTATIVE QUASIMARTINGALE SPACES
}

\author{
CONGBIAN MA* and YOULIANG HOU
}

Communicated by K. Zhu

\begin{abstract}
Let $\widehat{L}_{p}(\mathcal{M})$ be the space of bounded $L_{p}(\mathcal{M})$-quasimartingales. We prove that, with equivalent norms, $\left(\widehat{L}_{p_{0}}(\mathcal{M}), \widehat{L}_{p_{1}}(\mathcal{M})\right)_{\theta, p}=\widehat{L}_{p}(\mathcal{M})$, where $1<p_{0}, p_{1} \leq \infty, 1<\theta<1$, and $\frac{1}{p}=\frac{1-\theta}{p_{0}}+\frac{\theta}{p_{1}}$. We also prove that, for $1<p<$ $q<\infty,\left(\widehat{\mathrm{BMO}}^{c}(\mathcal{M}), \widehat{\mathcal{H}}_{p}^{c}(\mathcal{M})\right)_{\frac{p}{q}, q}=\widehat{\mathcal{H}}_{q}^{c}(\mathcal{M})$ and $\left(\widehat{\mathrm{BMO}}^{r}(\mathcal{M}), \widehat{\mathcal{H}}_{p}^{r}(\mathcal{M})\right)_{\frac{p}{q}, q}=$ $\widehat{\mathcal{H}}_{q}^{r}(\mathcal{M})$, where $\widehat{\mathcal{H}}_{p}(\mathcal{M})$ and $\widehat{\mathrm{BMO}}(\mathcal{M})$ are, respectively, the Hardy space and the bounded mean oscillation space of noncommutative quasimartingales.
\end{abstract}

\section{IntRoduction}

Inspired by quantum mechanics and probability, noncommutative probability has become an independent field of mathematical research. Today, many of the classical martingale inequalities have been transferred to the noncommutative setting. These include, in particular, the Doob maximal inequality, the BurkholderGundy inequality, several weak-type $(1,1)$ inequalities, and the Gundy decomposition.

As for the interpolation between the spaces of noncommutative martingales, we recall the formula on real and complex interpolation of noncommutative

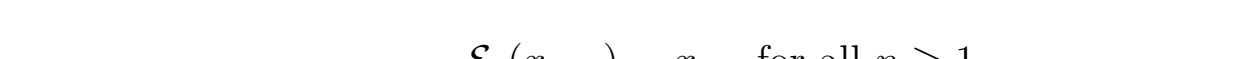

$$
\left(L_{p_{0}}(\mathcal{M}), L_{p_{1}}(\mathcal{M})\right)_{\theta, p}=L_{p}(\mathcal{M}) \quad \text { and } \quad\left(L_{p_{0}}(\mathcal{M}), L_{p_{1}}(\mathcal{M})\right)_{\theta}=L_{p}(\mathcal{M})
$$

Copyright 2016 by the Tusi Mathematical Research Group.

Received Dec. 2, 2015; Accepted Feb. 3, 2016.

${ }^{*}$ Corresponding author.

2010 Mathematics Subject Classification. Primary 46L53; Secondary 46L52, 60 G42.

Keywords. noncommutative quasimartingale, interpolation space, Hardy space. 


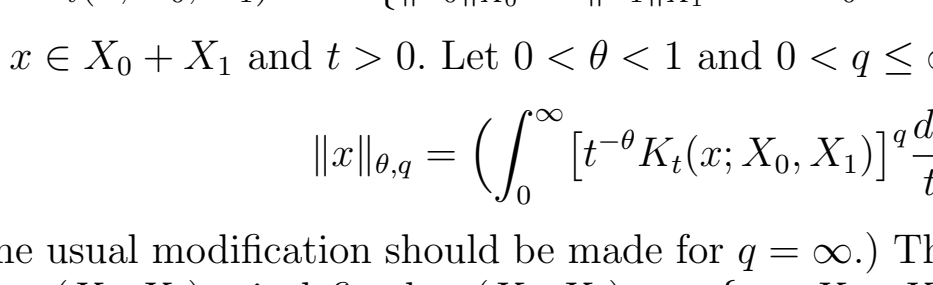

\title{
INTERPOLATION OF NONCOMMUTATIVE QUASIMARTINGALE SPACES
}

\author{
CONGBIAN MA* and YOULIANG HOU
}

Communicated by K. Zhu

\begin{abstract}
Let $\widehat{L}_{p}(\mathcal{M})$ be the space of bounded $L_{p}(\mathcal{M})$-quasimartingales. We prove that, with equivalent norms, $\left(\widehat{L}_{p_{0}}(\mathcal{M}), \widehat{L}_{p_{1}}(\mathcal{M})\right)_{\theta, p}=\widehat{L}_{p}(\mathcal{M})$, where $1<p_{0}, p_{1} \leq \infty, 1<\theta<1$, and $\frac{1}{p}=\frac{1-\theta}{p_{0}}+\frac{\theta}{p_{1}}$. We also prove that, for $1<p<$ $q<\infty,\left(\widehat{\mathrm{BMO}}^{c}(\mathcal{M}), \widehat{\mathcal{H}}_{p}^{c}(\mathcal{M})\right)_{\frac{p}{q}, q}=\widehat{\mathcal{H}}_{q}^{c}(\mathcal{M})$ and $\left(\widehat{\mathrm{BMO}}^{r}(\mathcal{M}), \widehat{\mathcal{H}}_{p}^{r}(\mathcal{M})\right)_{\frac{p}{q}, q}=$ $\widehat{\mathcal{H}}_{q}^{r}(\mathcal{M})$, where $\widehat{\mathcal{H}}_{p}(\mathcal{M})$ and $\widehat{\mathrm{BMO}}(\mathcal{M})$ are, respectively, the Hardy space and the bounded mean oscillation space of noncommutative quasimartingales.
\end{abstract}

\section{IntRoduction}

Inspired by quantum mechanics and probability, noncommutative probability has become an independent field of mathematical research. Today, many of the classical martingale inequalities have been transferred to the noncommutative setting. These include, in particular, the Doob maximal inequality, the BurkholderGundy inequality, several weak-type $(1,1)$ inequalities, and the Gundy decomposition.

As for the interpolation between the spaces of noncommutative martingales, we recall the formula on real and complex interpolation of noncommutative

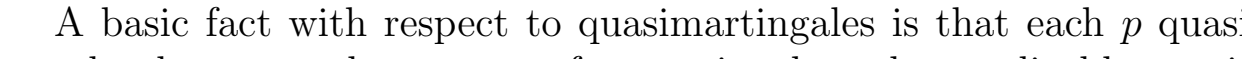

$$
\left(L_{p_{0}}(\mathcal{M}), L_{p_{1}}(\mathcal{M})\right)_{\theta, p}=L_{p}(\mathcal{M}) \quad \text { and } \quad\left(L_{p_{0}}(\mathcal{M}), L_{p_{1}}(\mathcal{M})\right)_{\theta}=L_{p}(\mathcal{M})
$$

Copyright 2016 by the Tusi Mathematical Research Group.

Received Dec. 2, 2015; Accepted Feb. 3, 2016.

${ }^{*}$ Corresponding author.

2010 Mathematics Subject Classification. Primary 46L53; Secondary 46L52, 60 G42.

Keywords. noncommutative quasimartingale, interpolation space, Hardy space. 


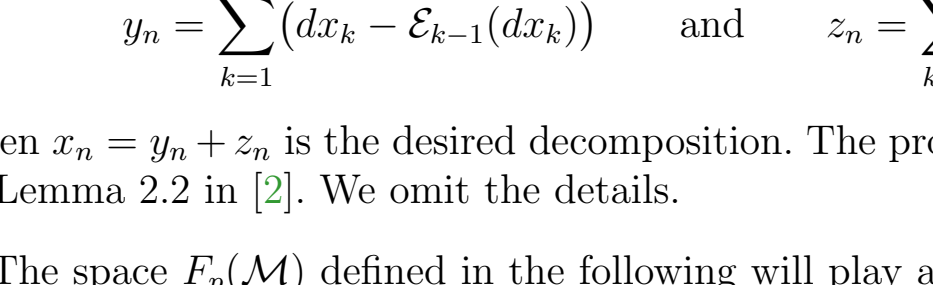

\title{
INTERPOLATION OF NONCOMMUTATIVE QUASIMARTINGALE SPACES
}

\author{
CONGBIAN MA* and YOULIANG HOU
}

Communicated by K. Zhu

\begin{abstract}
Let $\widehat{L}_{p}(\mathcal{M})$ be the space of bounded $L_{p}(\mathcal{M})$-quasimartingales. We prove that, with equivalent norms, $\left(\widehat{L}_{p_{0}}(\mathcal{M}), \widehat{L}_{p_{1}}(\mathcal{M})\right)_{\theta, p}=\widehat{L}_{p}(\mathcal{M})$, where $1<p_{0}, p_{1} \leq \infty, 1<\theta<1$, and $\frac{1}{p}=\frac{1-\theta}{p_{0}}+\frac{\theta}{p_{1}}$. We also prove that, for $1<p<$ $q<\infty,\left(\widehat{\mathrm{BMO}}^{c}(\mathcal{M}), \widehat{\mathcal{H}}_{p}^{c}(\mathcal{M})\right)_{\frac{p}{q}, q}=\widehat{\mathcal{H}}_{q}^{c}(\mathcal{M})$ and $\left(\widehat{\mathrm{BMO}}^{r}(\mathcal{M}), \widehat{\mathcal{H}}_{p}^{r}(\mathcal{M})\right)_{\frac{p}{q}, q}=$ $\widehat{\mathcal{H}}_{q}^{r}(\mathcal{M})$, where $\widehat{\mathcal{H}}_{p}(\mathcal{M})$ and $\widehat{\mathrm{BMO}}(\mathcal{M})$ are, respectively, the Hardy space and the bounded mean oscillation space of noncommutative quasimartingales.
\end{abstract}

\section{IntRoduction}

Inspired by quantum mechanics and probability, noncommutative probability has become an independent field of mathematical research. Today, many of the classical martingale inequalities have been transferred to the noncommutative setting. These include, in particular, the Doob maximal inequality, the BurkholderGundy inequality, several weak-type $(1,1)$ inequalities, and the Gundy decomposition.

As for the interpolation between the spaces of noncommutative martingales, we recall the formula on real and complex interpolation of noncommutative

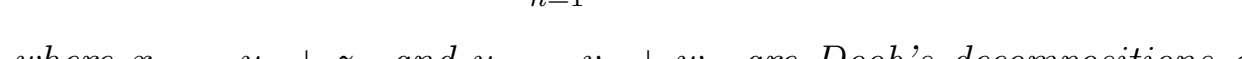

$$
\left(L_{p_{0}}(\mathcal{M}), L_{p_{1}}(\mathcal{M})\right)_{\theta, p}=L_{p}(\mathcal{M}) \quad \text { and } \quad\left(L_{p_{0}}(\mathcal{M}), L_{p_{1}}(\mathcal{M})\right)_{\theta}=L_{p}(\mathcal{M})
$$

Copyright 2016 by the Tusi Mathematical Research Group.

Received Dec. 2, 2015; Accepted Feb. 3, 2016.

${ }^{*}$ Corresponding author.

2010 Mathematics Subject Classification. Primary 46L53; Secondary 46L52, 60 G42.

Keywords. noncommutative quasimartingale, interpolation space, Hardy space. 


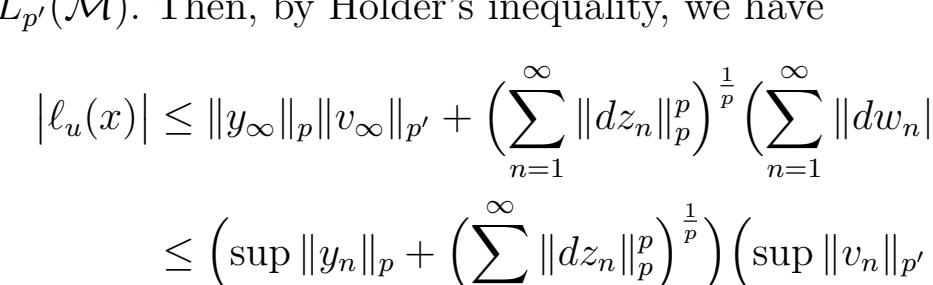

\title{
INTERPOLATION OF NONCOMMUTATIVE QUASIMARTINGALE SPACES
}

\author{
CONGBIAN MA* and YOULIANG HOU
}

Communicated by K. Zhu

\begin{abstract}
Let $\widehat{L}_{p}(\mathcal{M})$ be the space of bounded $L_{p}(\mathcal{M})$-quasimartingales. We prove that, with equivalent norms, $\left(\widehat{L}_{p_{0}}(\mathcal{M}), \widehat{L}_{p_{1}}(\mathcal{M})\right)_{\theta, p}=\widehat{L}_{p}(\mathcal{M})$, where $1<p_{0}, p_{1} \leq \infty, 1<\theta<1$, and $\frac{1}{p}=\frac{1-\theta}{p_{0}}+\frac{\theta}{p_{1}}$. We also prove that, for $1<p<$ $q<\infty,\left(\widehat{\mathrm{BMO}}^{c}(\mathcal{M}), \widehat{\mathcal{H}}_{p}^{c}(\mathcal{M})\right)_{\frac{p}{q}, q}=\widehat{\mathcal{H}}_{q}^{c}(\mathcal{M})$ and $\left(\widehat{\mathrm{BMO}}^{r}(\mathcal{M}), \widehat{\mathcal{H}}_{p}^{r}(\mathcal{M})\right)_{\frac{p}{q}, q}=$ $\widehat{\mathcal{H}}_{q}^{r}(\mathcal{M})$, where $\widehat{\mathcal{H}}_{p}(\mathcal{M})$ and $\widehat{\mathrm{BMO}}(\mathcal{M})$ are, respectively, the Hardy space and the bounded mean oscillation space of noncommutative quasimartingales.
\end{abstract}

\section{IntRoduction}

Inspired by quantum mechanics and probability, noncommutative probability has become an independent field of mathematical research. Today, many of the classical martingale inequalities have been transferred to the noncommutative setting. These include, in particular, the Doob maximal inequality, the BurkholderGundy inequality, several weak-type $(1,1)$ inequalities, and the Gundy decomposition.

As for the interpolation between the spaces of noncommutative martingales, we recall the formula on real and complex interpolation of noncommutative

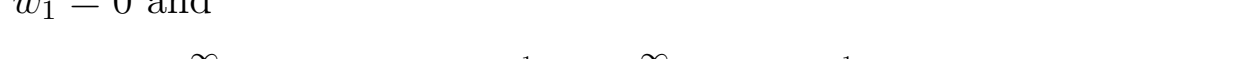

$$
\left(L_{p_{0}}(\mathcal{M}), L_{p_{1}}(\mathcal{M})\right)_{\theta, p}=L_{p}(\mathcal{M}) \quad \text { and } \quad\left(L_{p_{0}}(\mathcal{M}), L_{p_{1}}(\mathcal{M})\right)_{\theta}=L_{p}(\mathcal{M})
$$

Copyright 2016 by the Tusi Mathematical Research Group.

Received Dec. 2, 2015; Accepted Feb. 3, 2016.

${ }^{*}$ Corresponding author.

2010 Mathematics Subject Classification. Primary 46L53; Secondary 46L52, 60 G42.

Keywords. noncommutative quasimartingale, interpolation space, Hardy space. 


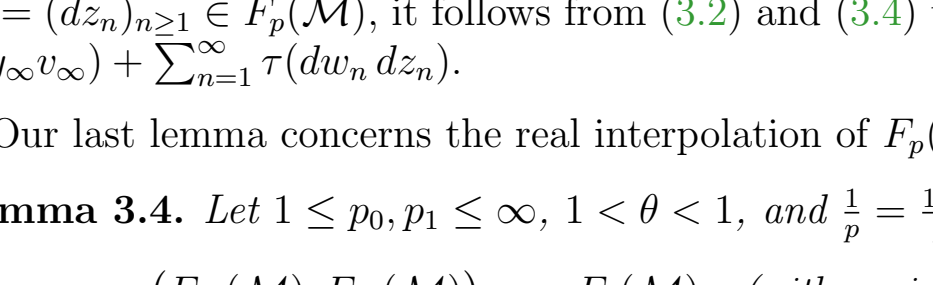

\title{
INTERPOLATION OF NONCOMMUTATIVE QUASIMARTINGALE SPACES
}

\author{
CONGBIAN MA* and YOULIANG HOU
}

Communicated by K. Zhu

\begin{abstract}
Let $\widehat{L}_{p}(\mathcal{M})$ be the space of bounded $L_{p}(\mathcal{M})$-quasimartingales. We prove that, with equivalent norms, $\left(\widehat{L}_{p_{0}}(\mathcal{M}), \widehat{L}_{p_{1}}(\mathcal{M})\right)_{\theta, p}=\widehat{L}_{p}(\mathcal{M})$, where $1<p_{0}, p_{1} \leq \infty, 1<\theta<1$, and $\frac{1}{p}=\frac{1-\theta}{p_{0}}+\frac{\theta}{p_{1}}$. We also prove that, for $1<p<$ $q<\infty,\left(\widehat{\mathrm{BMO}}^{c}(\mathcal{M}), \widehat{\mathcal{H}}_{p}^{c}(\mathcal{M})\right)_{\frac{p}{q}, q}=\widehat{\mathcal{H}}_{q}^{c}(\mathcal{M})$ and $\left(\widehat{\mathrm{BMO}}^{r}(\mathcal{M}), \widehat{\mathcal{H}}_{p}^{r}(\mathcal{M})\right)_{\frac{p}{q}, q}=$ $\widehat{\mathcal{H}}_{q}^{r}(\mathcal{M})$, where $\widehat{\mathcal{H}}_{p}(\mathcal{M})$ and $\widehat{\mathrm{BMO}}(\mathcal{M})$ are, respectively, the Hardy space and the bounded mean oscillation space of noncommutative quasimartingales.
\end{abstract}

\section{IntRoduction}

Inspired by quantum mechanics and probability, noncommutative probability has become an independent field of mathematical research. Today, many of the classical martingale inequalities have been transferred to the noncommutative setting. These include, in particular, the Doob maximal inequality, the BurkholderGundy inequality, several weak-type $(1,1)$ inequalities, and the Gundy decomposition.

As for the interpolation between the spaces of noncommutative martingales, we recall the formula on real and complex interpolation of noncommutative

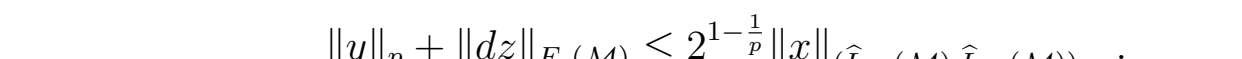

$$
\left(L_{p_{0}}(\mathcal{M}), L_{p_{1}}(\mathcal{M})\right)_{\theta, p}=L_{p}(\mathcal{M}) \quad \text { and } \quad\left(L_{p_{0}}(\mathcal{M}), L_{p_{1}}(\mathcal{M})\right)_{\theta}=L_{p}(\mathcal{M})
$$

Copyright 2016 by the Tusi Mathematical Research Group.

Received Dec. 2, 2015; Accepted Feb. 3, 2016.

${ }^{*}$ Corresponding author.

2010 Mathematics Subject Classification. Primary 46L53; Secondary 46L52, 60 G42.

Keywords. noncommutative quasimartingale, interpolation space, Hardy space. 


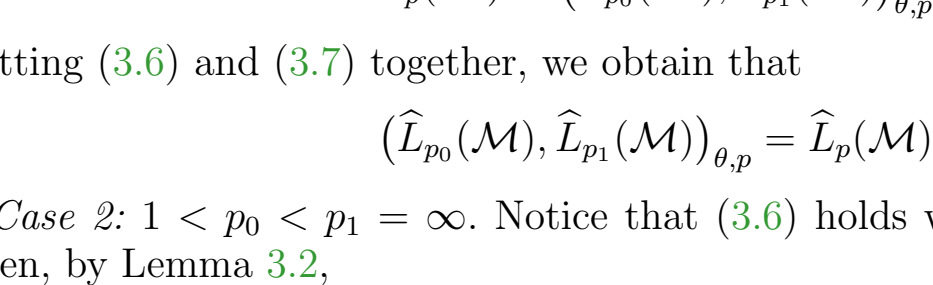

\title{
INTERPOLATION OF NONCOMMUTATIVE QUASIMARTINGALE SPACES
}

\author{
CONGBIAN MA* and YOULIANG HOU
}

Communicated by K. Zhu

\begin{abstract}
Let $\widehat{L}_{p}(\mathcal{M})$ be the space of bounded $L_{p}(\mathcal{M})$-quasimartingales. We prove that, with equivalent norms, $\left(\widehat{L}_{p_{0}}(\mathcal{M}), \widehat{L}_{p_{1}}(\mathcal{M})\right)_{\theta, p}=\widehat{L}_{p}(\mathcal{M})$, where $1<p_{0}, p_{1} \leq \infty, 1<\theta<1$, and $\frac{1}{p}=\frac{1-\theta}{p_{0}}+\frac{\theta}{p_{1}}$. We also prove that, for $1<p<$ $q<\infty,\left(\widehat{\mathrm{BMO}}^{c}(\mathcal{M}), \widehat{\mathcal{H}}_{p}^{c}(\mathcal{M})\right)_{\frac{p}{q}, q}=\widehat{\mathcal{H}}_{q}^{c}(\mathcal{M})$ and $\left(\widehat{\mathrm{BMO}}^{r}(\mathcal{M}), \widehat{\mathcal{H}}_{p}^{r}(\mathcal{M})\right)_{\frac{p}{q}, q}=$ $\widehat{\mathcal{H}}_{q}^{r}(\mathcal{M})$, where $\widehat{\mathcal{H}}_{p}(\mathcal{M})$ and $\widehat{\mathrm{BMO}}(\mathcal{M})$ are, respectively, the Hardy space and the bounded mean oscillation space of noncommutative quasimartingales.
\end{abstract}

\section{IntRoduction}

Inspired by quantum mechanics and probability, noncommutative probability has become an independent field of mathematical research. Today, many of the classical martingale inequalities have been transferred to the noncommutative setting. These include, in particular, the Doob maximal inequality, the BurkholderGundy inequality, several weak-type $(1,1)$ inequalities, and the Gundy decomposition.

As for the interpolation between the spaces of noncommutative martingales, we recall the formula on real and complex interpolation of noncommutative

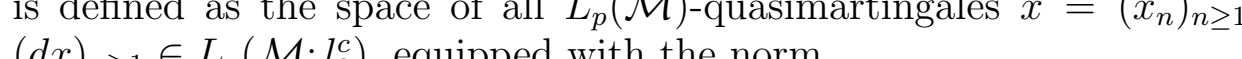

$$
\left(L_{p_{0}}(\mathcal{M}), L_{p_{1}}(\mathcal{M})\right)_{\theta, p}=L_{p}(\mathcal{M}) \quad \text { and } \quad\left(L_{p_{0}}(\mathcal{M}), L_{p_{1}}(\mathcal{M})\right)_{\theta}=L_{p}(\mathcal{M})
$$

Copyright 2016 by the Tusi Mathematical Research Group.

Received Dec. 2, 2015; Accepted Feb. 3, 2016.

${ }^{*}$ Corresponding author.

2010 Mathematics Subject Classification. Primary 46L53; Secondary 46L52, 60 G42.

Keywords. noncommutative quasimartingale, interpolation space, Hardy space. 


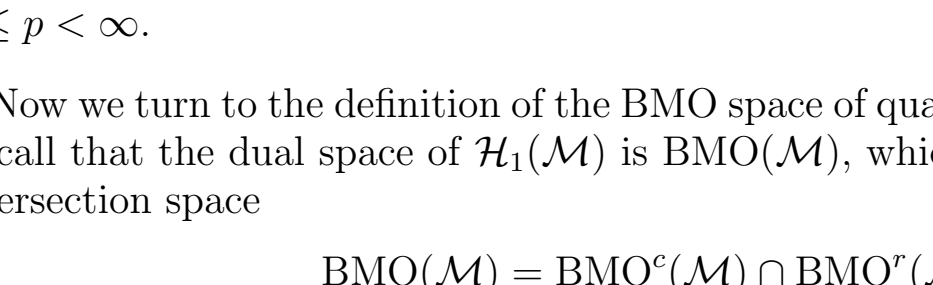

\title{
INTERPOLATION OF NONCOMMUTATIVE QUASIMARTINGALE SPACES
}

\author{
CONGBIAN MA* and YOULIANG HOU
}

Communicated by K. Zhu

\begin{abstract}
Let $\widehat{L}_{p}(\mathcal{M})$ be the space of bounded $L_{p}(\mathcal{M})$-quasimartingales. We prove that, with equivalent norms, $\left(\widehat{L}_{p_{0}}(\mathcal{M}), \widehat{L}_{p_{1}}(\mathcal{M})\right)_{\theta, p}=\widehat{L}_{p}(\mathcal{M})$, where $1<p_{0}, p_{1} \leq \infty, 1<\theta<1$, and $\frac{1}{p}=\frac{1-\theta}{p_{0}}+\frac{\theta}{p_{1}}$. We also prove that, for $1<p<$ $q<\infty,\left(\widehat{\mathrm{BMO}}^{c}(\mathcal{M}), \widehat{\mathcal{H}}_{p}^{c}(\mathcal{M})\right)_{\frac{p}{q}, q}=\widehat{\mathcal{H}}_{q}^{c}(\mathcal{M})$ and $\left(\widehat{\mathrm{BMO}}^{r}(\mathcal{M}), \widehat{\mathcal{H}}_{p}^{r}(\mathcal{M})\right)_{\frac{p}{q}, q}=$ $\widehat{\mathcal{H}}_{q}^{r}(\mathcal{M})$, where $\widehat{\mathcal{H}}_{p}(\mathcal{M})$ and $\widehat{\mathrm{BMO}}(\mathcal{M})$ are, respectively, the Hardy space and the bounded mean oscillation space of noncommutative quasimartingales.
\end{abstract}

\section{IntRoduction}

Inspired by quantum mechanics and probability, noncommutative probability has become an independent field of mathematical research. Today, many of the classical martingale inequalities have been transferred to the noncommutative setting. These include, in particular, the Doob maximal inequality, the BurkholderGundy inequality, several weak-type $(1,1)$ inequalities, and the Gundy decomposition.

As for the interpolation between the spaces of noncommutative martingales, we recall the formula on real and complex interpolation of noncommutative

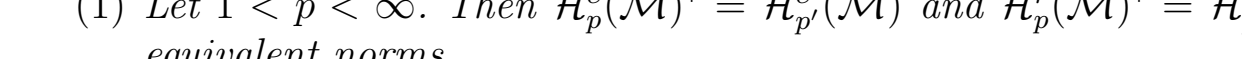

$$
\left(L_{p_{0}}(\mathcal{M}), L_{p_{1}}(\mathcal{M})\right)_{\theta, p}=L_{p}(\mathcal{M}) \quad \text { and } \quad\left(L_{p_{0}}(\mathcal{M}), L_{p_{1}}(\mathcal{M})\right)_{\theta}=L_{p}(\mathcal{M})
$$

Copyright 2016 by the Tusi Mathematical Research Group.

Received Dec. 2, 2015; Accepted Feb. 3, 2016.

${ }^{*}$ Corresponding author.

2010 Mathematics Subject Classification. Primary 46L53; Secondary 46L52, 60 G42.

Keywords. noncommutative quasimartingale, interpolation space, Hardy space. 


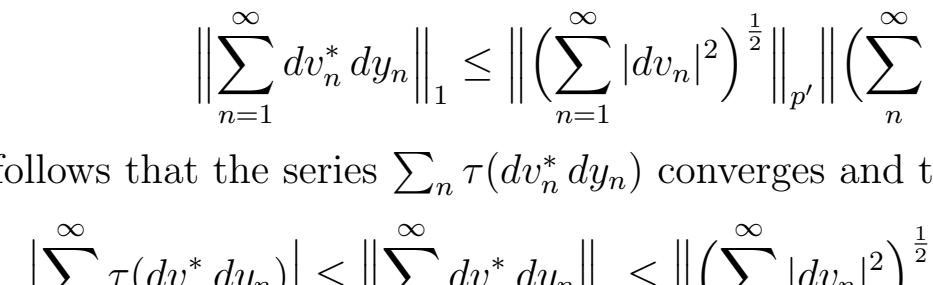

\title{
INTERPOLATION OF NONCOMMUTATIVE QUASIMARTINGALE SPACES
}

\author{
CONGBIAN MA* and YOULIANG HOU
}

Communicated by K. Zhu

\begin{abstract}
Let $\widehat{L}_{p}(\mathcal{M})$ be the space of bounded $L_{p}(\mathcal{M})$-quasimartingales. We prove that, with equivalent norms, $\left(\widehat{L}_{p_{0}}(\mathcal{M}), \widehat{L}_{p_{1}}(\mathcal{M})\right)_{\theta, p}=\widehat{L}_{p}(\mathcal{M})$, where $1<p_{0}, p_{1} \leq \infty, 1<\theta<1$, and $\frac{1}{p}=\frac{1-\theta}{p_{0}}+\frac{\theta}{p_{1}}$. We also prove that, for $1<p<$ $q<\infty,\left(\widehat{\mathrm{BMO}}^{c}(\mathcal{M}), \widehat{\mathcal{H}}_{p}^{c}(\mathcal{M})\right)_{\frac{p}{q}, q}=\widehat{\mathcal{H}}_{q}^{c}(\mathcal{M})$ and $\left(\widehat{\mathrm{BMO}}^{r}(\mathcal{M}), \widehat{\mathcal{H}}_{p}^{r}(\mathcal{M})\right)_{\frac{p}{q}, q}=$ $\widehat{\mathcal{H}}_{q}^{r}(\mathcal{M})$, where $\widehat{\mathcal{H}}_{p}(\mathcal{M})$ and $\widehat{\mathrm{BMO}}(\mathcal{M})$ are, respectively, the Hardy space and the bounded mean oscillation space of noncommutative quasimartingales.
\end{abstract}

\section{IntRoduction}

Inspired by quantum mechanics and probability, noncommutative probability has become an independent field of mathematical research. Today, many of the classical martingale inequalities have been transferred to the noncommutative setting. These include, in particular, the Doob maximal inequality, the BurkholderGundy inequality, several weak-type $(1,1)$ inequalities, and the Gundy decomposition.

As for the interpolation between the spaces of noncommutative martingales, we recall the formula on real and complex interpolation of noncommutative

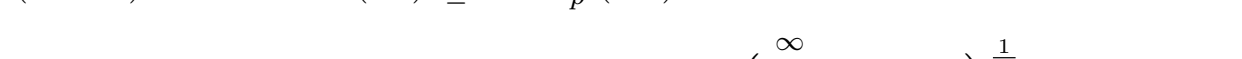

$$
\left(L_{p_{0}}(\mathcal{M}), L_{p_{1}}(\mathcal{M})\right)_{\theta, p}=L_{p}(\mathcal{M}) \quad \text { and } \quad\left(L_{p_{0}}(\mathcal{M}), L_{p_{1}}(\mathcal{M})\right)_{\theta}=L_{p}(\mathcal{M})
$$

Copyright 2016 by the Tusi Mathematical Research Group.

Received Dec. 2, 2015; Accepted Feb. 3, 2016.

${ }^{*}$ Corresponding author.

2010 Mathematics Subject Classification. Primary 46L53; Secondary 46L52, 60 G42.

Keywords. noncommutative quasimartingale, interpolation space, Hardy space. 


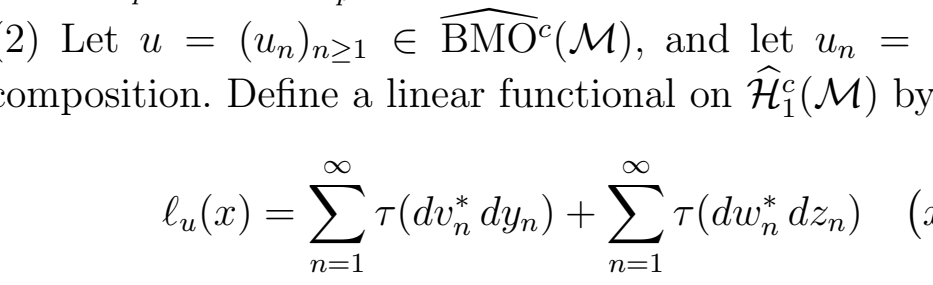

\title{
INTERPOLATION OF NONCOMMUTATIVE QUASIMARTINGALE SPACES
}

\author{
CONGBIAN MA* and YOULIANG HOU
}

Communicated by K. Zhu

\begin{abstract}
Let $\widehat{L}_{p}(\mathcal{M})$ be the space of bounded $L_{p}(\mathcal{M})$-quasimartingales. We prove that, with equivalent norms, $\left(\widehat{L}_{p_{0}}(\mathcal{M}), \widehat{L}_{p_{1}}(\mathcal{M})\right)_{\theta, p}=\widehat{L}_{p}(\mathcal{M})$, where $1<p_{0}, p_{1} \leq \infty, 1<\theta<1$, and $\frac{1}{p}=\frac{1-\theta}{p_{0}}+\frac{\theta}{p_{1}}$. We also prove that, for $1<p<$ $q<\infty,\left(\widehat{\mathrm{BMO}}^{c}(\mathcal{M}), \widehat{\mathcal{H}}_{p}^{c}(\mathcal{M})\right)_{\frac{p}{q}, q}=\widehat{\mathcal{H}}_{q}^{c}(\mathcal{M})$ and $\left(\widehat{\mathrm{BMO}}^{r}(\mathcal{M}), \widehat{\mathcal{H}}_{p}^{r}(\mathcal{M})\right)_{\frac{p}{q}, q}=$ $\widehat{\mathcal{H}}_{q}^{r}(\mathcal{M})$, where $\widehat{\mathcal{H}}_{p}(\mathcal{M})$ and $\widehat{\mathrm{BMO}}(\mathcal{M})$ are, respectively, the Hardy space and the bounded mean oscillation space of noncommutative quasimartingales.
\end{abstract}

\section{IntRoduction}

Inspired by quantum mechanics and probability, noncommutative probability has become an independent field of mathematical research. Today, many of the classical martingale inequalities have been transferred to the noncommutative setting. These include, in particular, the Doob maximal inequality, the BurkholderGundy inequality, several weak-type $(1,1)$ inequalities, and the Gundy decomposition.

As for the interpolation between the spaces of noncommutative martingales, we recall the formula on real and complex interpolation of noncommutative

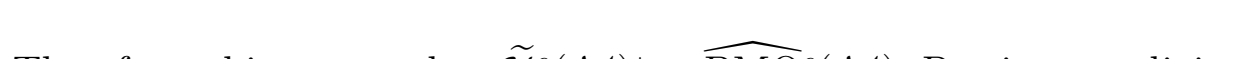

$$
\left(L_{p_{0}}(\mathcal{M}), L_{p_{1}}(\mathcal{M})\right)_{\theta, p}=L_{p}(\mathcal{M}) \quad \text { and } \quad\left(L_{p_{0}}(\mathcal{M}), L_{p_{1}}(\mathcal{M})\right)_{\theta}=L_{p}(\mathcal{M})
$$

Copyright 2016 by the Tusi Mathematical Research Group.

Received Dec. 2, 2015; Accepted Feb. 3, 2016.

${ }^{*}$ Corresponding author.

2010 Mathematics Subject Classification. Primary 46L53; Secondary 46L52, 60 G42.

Keywords. noncommutative quasimartingale, interpolation space, Hardy space. 


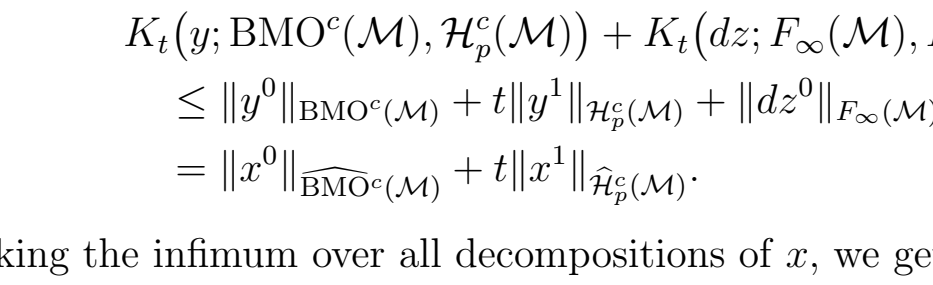

\title{
INTERPOLATION OF NONCOMMUTATIVE QUASIMARTINGALE SPACES
}

\author{
CONGBIAN MA* and YOULIANG HOU
}

Communicated by K. Zhu

\begin{abstract}
Let $\widehat{L}_{p}(\mathcal{M})$ be the space of bounded $L_{p}(\mathcal{M})$-quasimartingales. We prove that, with equivalent norms, $\left(\widehat{L}_{p_{0}}(\mathcal{M}), \widehat{L}_{p_{1}}(\mathcal{M})\right)_{\theta, p}=\widehat{L}_{p}(\mathcal{M})$, where $1<p_{0}, p_{1} \leq \infty, 1<\theta<1$, and $\frac{1}{p}=\frac{1-\theta}{p_{0}}+\frac{\theta}{p_{1}}$. We also prove that, for $1<p<$ $q<\infty,\left(\widehat{\mathrm{BMO}}^{c}(\mathcal{M}), \widehat{\mathcal{H}}_{p}^{c}(\mathcal{M})\right)_{\frac{p}{q}, q}=\widehat{\mathcal{H}}_{q}^{c}(\mathcal{M})$ and $\left(\widehat{\mathrm{BMO}}^{r}(\mathcal{M}), \widehat{\mathcal{H}}_{p}^{r}(\mathcal{M})\right)_{\frac{p}{q}, q}=$ $\widehat{\mathcal{H}}_{q}^{r}(\mathcal{M})$, where $\widehat{\mathcal{H}}_{p}(\mathcal{M})$ and $\widehat{\mathrm{BMO}}(\mathcal{M})$ are, respectively, the Hardy space and the bounded mean oscillation space of noncommutative quasimartingales.
\end{abstract}

\section{IntRoduction}

Inspired by quantum mechanics and probability, noncommutative probability has become an independent field of mathematical research. Today, many of the classical martingale inequalities have been transferred to the noncommutative setting. These include, in particular, the Doob maximal inequality, the BurkholderGundy inequality, several weak-type $(1,1)$ inequalities, and the Gundy decomposition.

As for the interpolation between the spaces of noncommutative martingales, we recall the formula on real and complex interpolation of noncommutative

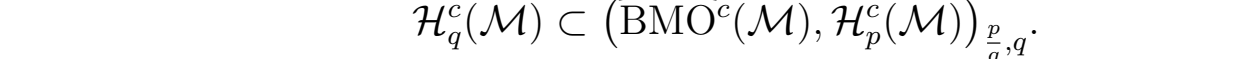

$$
\left(L_{p_{0}}(\mathcal{M}), L_{p_{1}}(\mathcal{M})\right)_{\theta, p}=L_{p}(\mathcal{M}) \quad \text { and } \quad\left(L_{p_{0}}(\mathcal{M}), L_{p_{1}}(\mathcal{M})\right)_{\theta}=L_{p}(\mathcal{M})
$$

Copyright 2016 by the Tusi Mathematical Research Group.

Received Dec. 2, 2015; Accepted Feb. 3, 2016.

${ }^{*}$ Corresponding author.

2010 Mathematics Subject Classification. Primary 46L53; Secondary 46L52, 60 G42.

Keywords. noncommutative quasimartingale, interpolation space, Hardy space. 


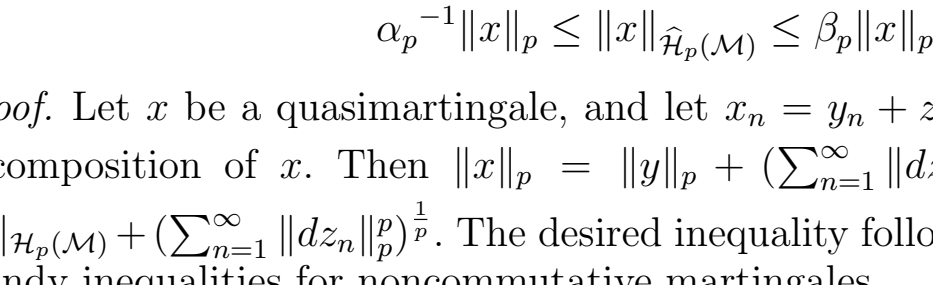

\title{
INTERPOLATION OF NONCOMMUTATIVE QUASIMARTINGALE SPACES
}

\author{
CONGBIAN MA* and YOULIANG HOU
}

Communicated by K. Zhu

\begin{abstract}
Let $\widehat{L}_{p}(\mathcal{M})$ be the space of bounded $L_{p}(\mathcal{M})$-quasimartingales. We prove that, with equivalent norms, $\left(\widehat{L}_{p_{0}}(\mathcal{M}), \widehat{L}_{p_{1}}(\mathcal{M})\right)_{\theta, p}=\widehat{L}_{p}(\mathcal{M})$, where $1<p_{0}, p_{1} \leq \infty, 1<\theta<1$, and $\frac{1}{p}=\frac{1-\theta}{p_{0}}+\frac{\theta}{p_{1}}$. We also prove that, for $1<p<$ $q<\infty,\left(\widehat{\mathrm{BMO}}^{c}(\mathcal{M}), \widehat{\mathcal{H}}_{p}^{c}(\mathcal{M})\right)_{\frac{p}{q}, q}=\widehat{\mathcal{H}}_{q}^{c}(\mathcal{M})$ and $\left(\widehat{\mathrm{BMO}}^{r}(\mathcal{M}), \widehat{\mathcal{H}}_{p}^{r}(\mathcal{M})\right)_{\frac{p}{q}, q}=$ $\widehat{\mathcal{H}}_{q}^{r}(\mathcal{M})$, where $\widehat{\mathcal{H}}_{p}(\mathcal{M})$ and $\widehat{\mathrm{BMO}}(\mathcal{M})$ are, respectively, the Hardy space and the bounded mean oscillation space of noncommutative quasimartingales.
\end{abstract}

\section{IntRoduction}

Inspired by quantum mechanics and probability, noncommutative probability has become an independent field of mathematical research. Today, many of the classical martingale inequalities have been transferred to the noncommutative setting. These include, in particular, the Doob maximal inequality, the BurkholderGundy inequality, several weak-type $(1,1)$ inequalities, and the Gundy decomposition.

As for the interpolation between the spaces of noncommutative martingales, we recall the formula on real and complex interpolation of noncommutative

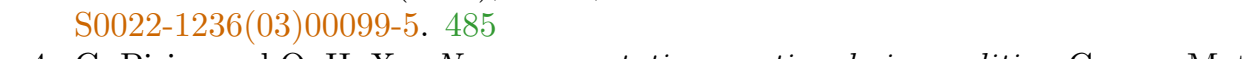

$$
\left(L_{p_{0}}(\mathcal{M}), L_{p_{1}}(\mathcal{M})\right)_{\theta, p}=L_{p}(\mathcal{M}) \quad \text { and } \quad\left(L_{p_{0}}(\mathcal{M}), L_{p_{1}}(\mathcal{M})\right)_{\theta}=L_{p}(\mathcal{M})
$$

Copyright 2016 by the Tusi Mathematical Research Group.

Received Dec. 2, 2015; Accepted Feb. 3, 2016.

${ }^{*}$ Corresponding author.

2010 Mathematics Subject Classification. Primary 46L53; Secondary 46L52, 60 G42.

Keywords. noncommutative quasimartingale, interpolation space, Hardy space. 